\title{
Unveiling the nature of extragalactic jets with Chandra observations
}

\author{
F. Massaro ${ }^{1}$, C. C. Cheung ${ }^{2}$ and D. E. Harris ${ }^{1}$ \\ ${ }^{1}$ Harvard - Smithsonian Astrophysical Observatory, Center for Astrophysics \\ 60 Garden Street, Cambridge, MA 02138, USA \\ email: fmassaro@head.cfa.harvard.edu \\ ${ }^{2}$ NRL/NRC, 4555 Overlook Ave SW, Washington, DC, 20375 USA
}

\begin{abstract}
In 1974 Fanaroff \& Riley divided the extended radio sources into two classes, on the basis of their radio morphology and power. For several years we have been collecting basic parameters for extragalactic jets detected in the X-rays, looking for an extension of the classification criterion, based on their radio and X-rays properties. The fact that different processes have been proposed to explain their X-ray radiation, (synchrotron vs inverse Compton emission) suggests the possibility of a new classification scheme. However, comparing the radio-to-X-ray properties of the extragalactic jets, several aspects on their nature became unexpectedly unclear.
\end{abstract}

Keywords. radiation mechanisms: non-thermal, X-rays: galaxies, galaxies: active, galaxies: jets.

\section{Introduction}

Radio galaxies and Quasars show a small number of features: jets, hotspots, and lobes. Following the definition of Bridle (1986), jets are highly collimated structures, distinguishable from other extended components either spatially or by brightness contrast, starting in the central region of the host active galactic nucleus (AGN). As suggested by Leahy et al. (1997), hotspots are brightness peaks which are neither a core nor part of a jet, usually lying where the jet terminates. Lobes are extended components on kpc scale, typically double, roughly symmetrical and ellipsoidal lying on both sides of the host radio galaxy or quasar.

In 1974, it was first noticed by Fanaroff and Riley that the relative positions of regions of high and low surface brightness in the extended regions of radio galaxies are correlated with their radio luminosity (central bright=class I vs. edge bright=class II). They found that nearly all sources with luminosity $L_{178 M \mathrm{H} z} \leqslant 2 \times 10^{25} h_{100}^{-2} \mathrm{~W} \mathrm{~Hz}^{-1} \mathrm{str}^{-1}$ were of Class I (i.e. FR I) while the brighter sources were nearly all of Class II (i.e. FR II).

The radio galaxy morphology turns out to reflect the method of energy transport in the radio source. FR I radio galaxies typically have bright jets in the centre, while FR IIs have faint jets but bright hotspots at the ends of their lobes (e.g. Baum et al. 1995). In particular, FR Is show surface brightness higher toward their cores while FR IIs toward their edges at radio frequencies.

The radio to optical emission arising from extended structures of radio galaxies and quasars is widely interpreted as synchrotron radiation by relativistic particles whereas the origin of X-ray emission in extended structures (jets and hotspots) is still unclear, but is certainly non-thermal. The main dichotomy lies in which mechanism, synchrotron or inverse Compton (IC) scattering, dominates the X-ray emission of jets. The former describes emission from low power jets, typically FR I, while the latter provides a good explanation for high power radio galaxy (i.e. FR II) and quasars. 
In the past ten years, we have collected a sample of radio galaxies and quasars for which the X-ray emission associated with their radio jets or hotspots has been detected by Chandra. The main goal of this project (hereinafter XJET project, Harris et al. 2010) is the development of a new classification criterion based not only on the radio morphology and power but also on the X-ray properties of these extended structures. We aim to build a method to distinguish between knots in jets and hotspots, both in radio galaxies and quasars, linked with the radiative process responsible for their radio and X-ray emission.

The list and all the basic informations on the sources in our sample (e.g. coordinates, redshift, radio classification etc.) can be found on the XJET webpage†. Our sample consists of 96 sources for a total of 194 extended components.

\section{X-ray Data reduction}

The X-ray data reduction has been performed following the standard reduction procedure described in the Chandra Interactive Analysis of Observations (CIAO) threads $\ddagger$, using CIAO v4.2 and the Chandra Calibration Database (CALDB) version 4.2.

Level 2 event files were generated using the acis_process_events task, after removing the hot pixels with acis_run_hotpix. Events were filtered for grades 0,2,3,4,6 and we removed pixel randomization.

Lightcurves for every dataset were extracted and checked for high background intervals, that have been eventually removed according to the criteria described in Chandra Interactive Analysis of Observations (CIAO) threads.

Astrometric registration was achieved by changing the appropriate keywords in the fits header so as to align the nuclear X-ray position with that of the radio.

We created 3 different fluxmaps (soft, medium, hard, in the ranges $0.5-1,1-2,2$ $-7 \mathrm{keV}$, respectively) by dividing the data with monochromatic exposure maps (with nominal energies of $\mathrm{soft}=0.75 \mathrm{keV}$, medium $=1.4 \mathrm{keV}$, and hard $=4 \mathrm{keV}$ ). The exposure maps and the flux maps were regridded to a common pixel size which was usually $1 / 4$ the size of a native ACIS pixel (native $=0.492^{\prime \prime}$ ). For sources of large angular extent we used $1 / 2$ or no regridding. To obtain maps with brightness units of ergs $\mathrm{cm}^{-2} \mathrm{~s}^{-1} \mathrm{pixel}^{-1}$, we multiplied each event by the nominal energy of its respective band.

To measure observed fluxes we construct appropriate regions (usually circular) as well as two adjacent background regions of the same size. We then measure the net flux in each region and in each energy band. After applying a small correction which is the ratio of the mean energy of the events within the 'on' aperture to the nominal energy applied earlier to all events, a one $\sigma$ error is assigned based on the usual $\sqrt{\text { number }- \text { of }- \text { counts }}$ in the on and background regions.

\section{Data analysis}

We developed two main tools to investigate the radio and the X-ray emission of the selected jets and hotspots.

By measuring the radio fluxes, using the radio maps available in the public archives (e.g. NVSS, NED) or kindly provided by our colleagues, we calculated the X-ray-to-radio flux ratio $\rho$, where the X-ray fluxes have been derived directly from our fluxmaps analysis. On the other hand, both the X-ray and the radio luminosities, $L_{X}$ and $L_{R}$, for the whole

$\dagger($ http : //hea - www.harvard.edu/XJET/)

$\ddagger($ http ://cxc.harvard.edu/ciao/guides/index.html) 

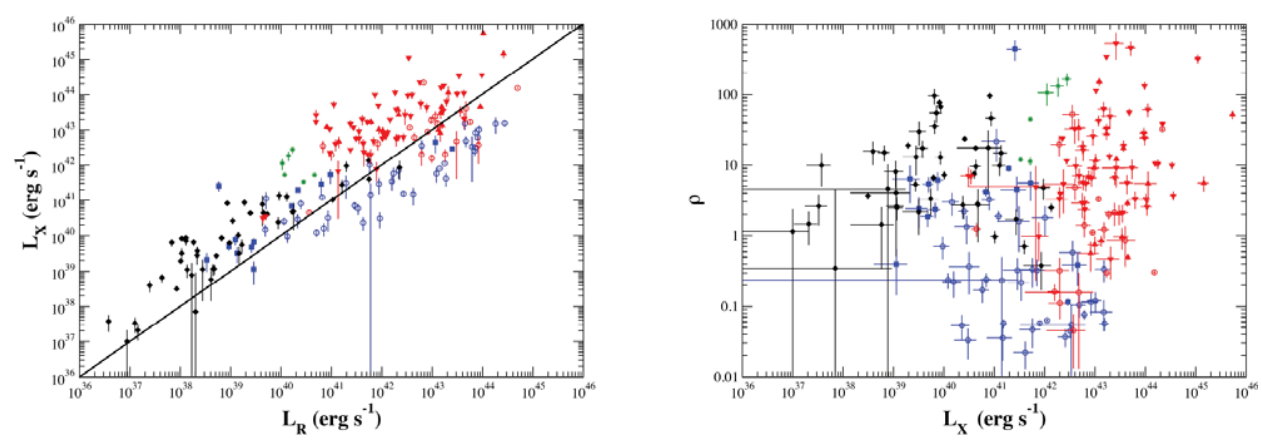

Figure 1. a - Left panel) The X-ray luminosity vs the radio luminosity of all the knots and hotspots in the selected radio galaxies and quasars. b - Right panel) The luminosity ratio vs the X-ray luminosity of the selected knots and hotspots. The nomenclature here reported has been adopted for all the plots in the paper, indicating: FR I knots (filled blue diamonds), FR II knots (filled blue squares), CDQ and LDQ knots (filled red triangles), BL Lac knots (green stars), FR II hotspots (i.e. hs2, open blue circles), LDQ hotspots (open red cricles).

sample of extended components (i.e. knots and hotspots), in radio galaxies and quasars, are plotted in Fig. 1a.

In addition, we also evaluated the X-ray hardness ratio $\mathrm{HR}$ using the hard, $H$, and the medium, $M$, X-ray fluxes by using the following relation: $(H-M) /(H+M)$. We neglected the soft X-ray flux in the estimate of HR because uncertain absorption corrections are much larger for the soft band than for the medium and hard bands. The hardness ratio HR has been used as a good indicator of the X-ray spectral index $\alpha_{X}$ of our extended components, because the short exposure of the available X-ray observations combined with their low intrinsic flux (i.e. $\sim 10^{-15} \mathrm{erg} \mathrm{s}^{-1} \mathrm{~cm}^{-2}$ ) do not allow us to estimate $\alpha_{X}$ precisely for the whole sample. We also note that because HRs are evaluated using X-ray fluxes there is a 1-1 relationship between $\alpha_{X}$ and HR.

We compare all the values of $\rho$ and HR dividing the knots and the hotspots of our sources in 5 categories as defined in the following. The nomenclature chosen has been adopted for all the figures. We considered knots in FR Is (i.e. k1, filled blue diamonds) and in FR IIs (i.e. k2, filled blue squares), knots in quasars (i.e. kq, filled red triangles) and in BL Lacs (i.e. kbl, green stars), while for the hotspots we defined those in FR IIs (i.e. hs2, open blue circles) and in LDQs (i.e. hsq, open red cricles).

All the main results derived form our analysis comparing the $\rho$ and the HR values of the different extended structure here defined are reported in the next section.

\section{Results}

The main observational results arising from our analysis are here summarized.

- The X-ray luminosities of knots in the BL Lac jets lie between FRI and Quasars (e.g. Fig. 1, 2a).

- Hotspots typically do not show luminosity ratios higher than 10 (e.g. Fig. 1, 2a).

- All knots have luminosity ratios higher 0.1 (e.g. Fig. 1, 2a).

- Knots of radio galaxies and quasars have similar luminosity ratio distributions (e.g. Fig. 1, 2).

- The distribution of the luminosity ratios, both for hotspots and knots, are similar between radio galaxies and quasars. This is a surprising point because, following the general interpretation, the X-ray emission arising from these extended structures has a 

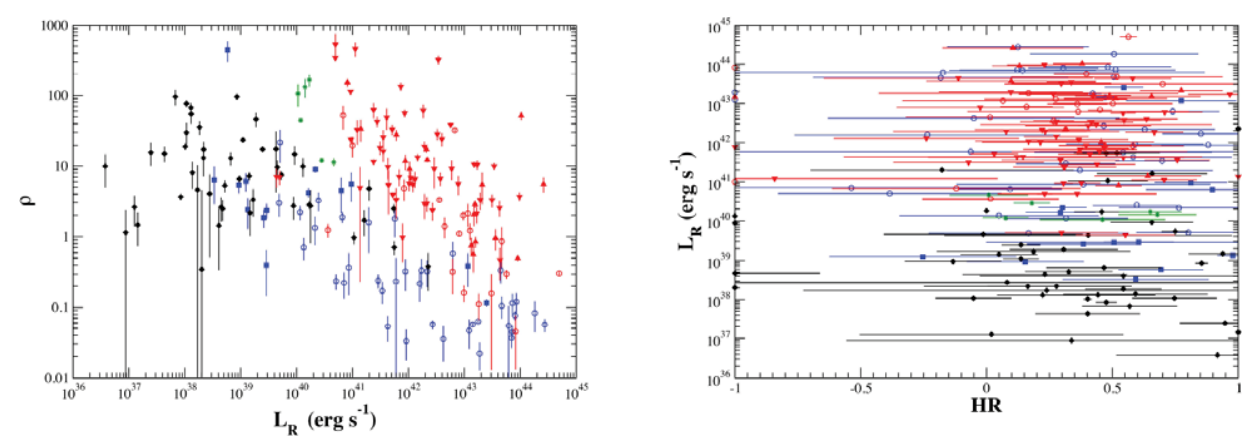

Figure 2. a - Left panel) The luminosity ratio vs the radio luminosity of the selected knots and hotspots. $\mathrm{b}$ - Right panel) The radio luminosity $v s$ the X-ray hardness ratios.

different origin (synchrotron $v s$ inverse Compton processes). So it was expected to find some differences between their luminosity ratio distributions.

- There are no differences between knots of quasars and radio galaxies in terms of HR (e.g. Fig. 2b).

- There are no differences between the HRs in hotspots of quasars and radio galaxies (e.g. Fig. 2b).

Consequently, the main conclusion arising from our analysis is that, despite the three radiative processes proposed to interpret the X-ray emission in knots and hotspots of both radio galaxies and quasars suggesting a different distributions of luminosity ratios and X-ray hardness ratios, no difference have been found.

\section{Acknowledgements}

F. Massaro acknowledges the Foundation BLANCEFLOR Boncompagni-Ludovisi, n'ee Bildt for the grant awarded him in 2010 to support his research. The work at SAO is supported by the NASA grant NNX10AD50G.

\section{References}

Fanaroff, B. L. \& Riley J. M. 1974, MNRAS, 167, 31

Bridle, A. H. 1986. Extragalactic jets - Trends and correlations. Can. J. Phys., 64, 353

Leahy, J. P. et al. 1997 MNRAS, 291, 20

Baum, S. A., Zirbel, E. L., \& O'Dea, C. P. 1995 ApJ, 451, 88

Harris, D. E., Massaro, F., \& Cheung, C. C. 2010, AIPC. 1248, 355

\section{Discussion}

SAMBRUnA: A synchrotron counterpart can be present in a QSR knot contributing to $\mathrm{X}$-ray emission, that HR may miss.

MASSARO: Unfortunately, the low statistic, due to short exposure of many of the considered Chandra observation do not allow us to distinguish between single and multicomponent models. So, it is certainly true that a second component may affect the estimates of the hardness ratios in the X-rays. 